\title{
Immunology of Transplant Protocols
}

\author{
Brian Gallay • Angelo DeMattos
}

Published online: 8 July 2014

(c) Springer Science+Business Media New York 2014

\begin{abstract}
The development of immunosuppressive medications to control the alloimmune response against donor Human leukocyte antigens (HLA) has permitted the transplantation of many solid organs as well as the treatment of graft versus host disease in bone marrow transplantation. The experience gained with these now routine therapies for end stage organ disease has led to protocols for composite tissue transplantation with grafts containing multiple organ types, such as skin, muscle, nerve, and bone. Successful transplant of limbs, face, and larynx has ushered in a new stage in the progress of clinical transplantation with identification of newly characterized interactions between the immune system and the allograft. The expanding number of clinically successful composite tissue transplants has increased the likelihood for subspecialists not in the traditional fields of nephrology, hepatology, pulmonology, or cardiology to encounter patients taking immunosuppressive medications. Therefore, it is useful to have a basic understanding of the rationale for and complications of therapies including these medications.
\end{abstract}

Keywords Laryngeal transplant - Immunosuppression protocol - Alloimmune response - Mixed chimerism . Composite tissue transplant

B. Gallay $(\bowtie) \cdot$ A. DeMattos

Transplant Nephrology Section, Davis School of Medicine, University of California, 2019 Housestaff Facility Building, 2315 Stockton Boulevard, Sacramento, CA 95817, USA e-mail: brian.gallay@ucdmc.ucdavis.edu

A. DeMattos

e-mail: angelo.demattos@ucdmc.ucdavis.edu

\section{Introduction}

Since the first clinically successful kidney transplant in 1954 [1], advances in surgical techniques and immunosuppressive therapies have made possible the routine treatment of end stage organ disease, including chronic kidney disease, cirrhosis, diabetes, pulmonary disease, and heart failure [2••]. These successes have prompted the development of protocols for vascularized composite tissue allotransplants (VCA), including limb, face, and laryngeal transplant. A novel feature of VCA is the multiple organ types involved, including skin, nerve, muscle, bone, and bone marrow. The immunogenicity of these tissues differs from that of solid organs, and therefore, the optimal immunosuppressive therapy is yet unknown. Nonetheless, the types of immunosuppression used for solid organ transplant have been successfully adapted for composite tissue allografts. The number of patients seen in clinical practice with VCA is likely to increase with time, and a larger group of subspecialists will need to be aware of the fundamental aspects of immunosuppression and allograft maintenance. Successful laryngeal transplantation, first in 1998 [3, 4•] and again in 2010 [5], has added an exciting new treatment modality. This review attempts to provide a basic knowledge of alloimmunity, acute and chronic graft rejection, and immunosuppressive therapy to prevent these complications. In addition, the intriguing prospect of developing immune tolerance protocols in composite tissue transplant is discussed.

\section{The Alloimmune Response}

The recipient immune response to alloantigens, primarily Human leukocyte antigens (HLA) expressed on donor 


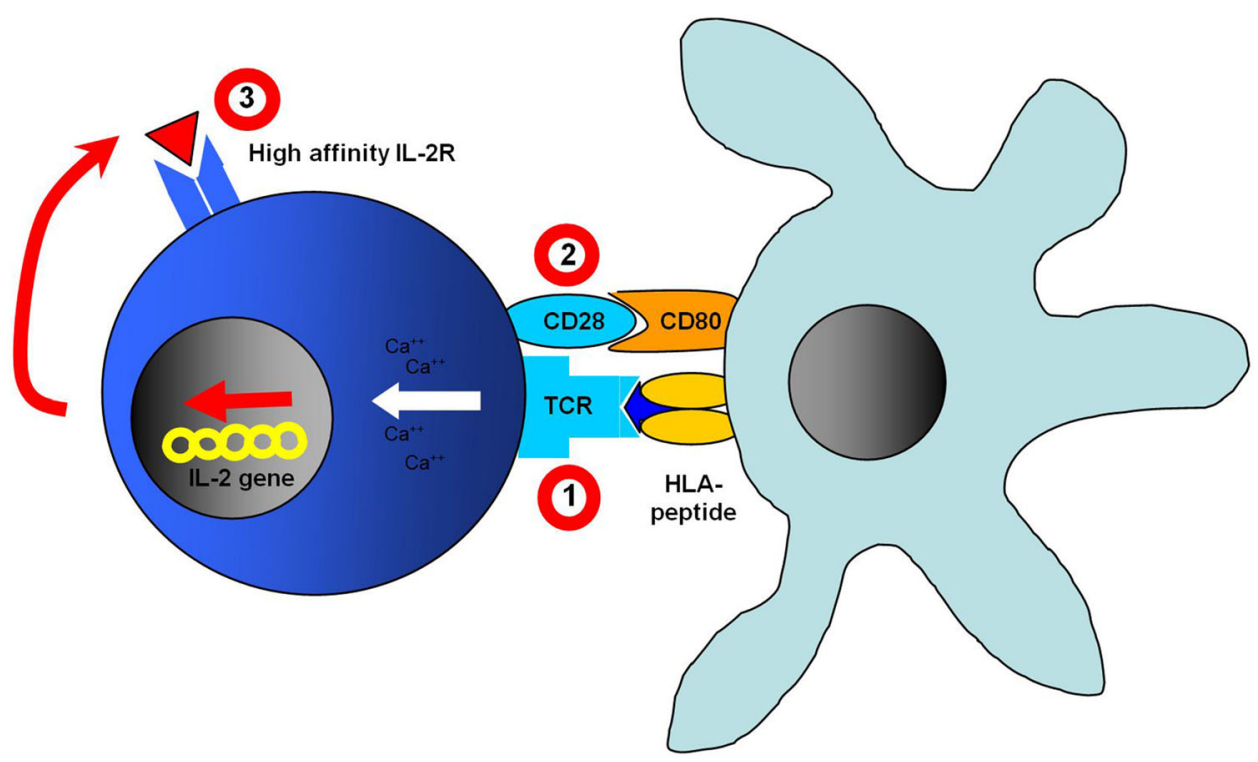

Fig. 1 T lymphocyte activation and targets for immunosuppression. The $\mathrm{T}$ cell receptor (TCR) engages alloantigen peptide presented by an HLA molecule on the surface of the antigen-presenting cell (APC). If the interaction is of sufficient affinity, then a TCR-HLA/peptide complex is formed, generating signal 1 . This interaction is stabilized and the signal amplified by the interaction of the costimulatory molecule CD28 with its ligand expressed by the APC or signal 2. The stable complex triggers release of calcium from intracellular stores, which activates several downstream functions, including transcription of T cell activation factors such as IL-2 and its high affinity receptor. Ligation of IL-2 with its receptor provides signal 3, which triggers

cells, includes both naïve and memory $\mathrm{T}$ lymphocytes. These lymphocytes express $\mathrm{T}$ cell receptors (TCR) which recognize specific peptide antigens exhibited on the surface of antigen presenting cells (APC) such as dendritic cells, macrophage, and B lymphocytes. APCs process donorderived proteins into peptides that are loaded onto class II and class II HLA molecules for presentation to $\mathrm{CD}^{+}$ (helper) and $\mathrm{CD}^{+}$(cytotoxic) $\mathrm{T}$ lymphocytes, respectively. The TCR-HLA/peptide interaction, or "signal 1", requires a costimulatory "signal 2 " for proper $\mathrm{T}$ cell activation and cytokine production to occur. Activated $\mathrm{CD} 4^{+}$ cells produce interleukin-2 (IL-2) which is critical for amplification of the $\mathrm{T}$ cell response through its autocrine and paracrine binding to high affinity IL-2 receptor or "signal 3" [6•, 7•] (Fig. 1).

Antigen presentation can occur within the allograft itself or within sites of the secondary immune system such as regional lymph nodes and spleen. $\mathrm{T}$ lymphocytes activated after alloantigen presentation circulate through the body and are targeted to areas of tissue injury and inflammation, such as the allograft in the postoperative period. Macrophage resident in the allograft produces chemotactic signals to attract $\mathrm{T}$ lymphocytes, neutrophils, and monocytes, while adhesion molecules expressed on the surface of graft further T cell entry into the cell cycle and proliferation. Immunosuppressive agents impair signal 1 by removing TCR from the surface of the $\mathrm{T}$ cell or depleting $\mathrm{T}$ cells (rabbit antithymocyte globulin, alemtuzumab), signal 2 by interfering with CD28-CD80 costimulation (belatacept), and signal 3 by decreasing production of IL-2 (calcineurin inhibitors) and blocking or removing high affinity IL-2 receptor from the surface of the $\mathrm{T}$ cell (basiliximab). Sirolimus prevents the activated $\mathrm{T}$ cell from entering the cell cycle, and antimetabolites (azathioprine, mycophenolate mofetil) inhibit proliferation of $\mathrm{T}$ cells that have entered the cell cycle

endothelium tether the circulating cells to the endothelial cell surface. This interaction is followed by stronger attachment to endothelial cells by other adhesion molecules and subsequent translocation between endothelial cells into the graft. After encountering target antigens, cytotoxic $\mathrm{CD}^{+}$cells release perforins, granzyme $\mathrm{B}$, and granulysin into donor cells, causing necrosis by intracellular injury to protein, nucleic acid, and lipids. In addition, binding of Fas ligand and other signals produced by cytotoxic $\mathrm{T}$ cells causes apoptotic death. Helper $\mathrm{CD} 4^{+}$cells enhance activation of cytotoxic $\mathrm{T}$ cells through production of soluble cytokines including IL-2 and of B lymphocytes by direct interaction with B cell immunoglobulin receptors that recognize donor-specific antigens. This direct contact interaction activates B lymphocytes to produce donorspecific antibodies which cause injury to the allograft by fixation of complement causing cell lysis and by antibodydependent cytotoxicity. The profile of cytokine production by $\mathrm{CD}^{+}$cells is also influenced by components of the native immune system including neutrophils, macrophage, and complement which recognize tissue injury and mount a stereotyped response. Depending on the cytokines produced, $\mathrm{T}$ lymphocytes may assume either an effector or regulatory role $[8 \cdot]$. 
Activation of the alloimmune response in solid organ transplants leads to acute cellular rejection, which is characterized histologically by inflammatory infiltrates of $\mathrm{T}$ lymphocytes, B lymphocytes, and often plasma cells, neutrophils, and eosinophils, causing active damage to specific organ structures. Disruption of renal tubular epithelium characterizes acute renal cellular rejection $[9,10]$, whereas injury to biliary ductal epithelium occurs in liver acute cellular rejection [11,12], and injury to endothelium, ductules, and acini is evident in pancreas acute cellular rejection [13]. Necrosis of cardiomyocytes is noted in heart transplant [14], and disruption of vascular endothelium and bronchiolar epithelium occurs in lung transplants [15]. Acute rejection of small bowel is characterized by epithelial injury, lamina propria inflammation, and crypt apoptotic body formation [16].

Due to the lack of adequate maintenance immunosuppression targeting B lymphocytes and plasma cells, acute antibody-mediated rejection (AMR) is often a more serious complication than cellular rejection. Donor-specific antibodies may be present at the time of transplant or may develop after allosensitization, but their contribution to allograft injury may not be readily detectable if only low present at low titer. Antibody binding causes graft endothelial cell injury by multiple mechanisms. It activates complement with subsequent cell lysis by the insertion of the C5b-9 membrane attack complex. Binding of antibodyendothelial cell complexes to the $\mathrm{Fc}$ receptors on the surface of effector cells such as macrophage, monocytes, and natural killer cells activates the effector to directly cause endothelial injury and lysis (antibody-mediated cytotoxicity, or ADCC). Finally, antibody binding may activate the cell surface expression of class I and class II HLA molecules which render the endothelial cell more susceptible to immune recognition [7•, 17, 18]. Histologic findings associated with AMR include intravascular microthrombus formation, fibrin deposition, and endothelial necrosis [19, 20].

Chronic rejection of solid organ transplants may develop from months to years after transplant and is generally characterized by injury to hollow structures. Small arteriolar vasculopathy and glomerulopathy occur in kidney, small airway fibrosis in lung (bronchiolitis obliterans), biliary duct loss and obstructive vasculopathy in liver, and coronary artery disease with concentric intravascular occlusion in heart transplant [9, 13, 21-23]. Chronic rejection leads to ischemia and irreversible fibrosis with ultimate graft loss. Although immunosuppression has been successful in decreasing the occurrence of acute rejection in solid organ transplant, long-term graft survival has not significantly improved due to the inability to control chronic rejection.

\section{Immunosuppressive Medications}

Clinical immunosuppression is targeted primarily against the $\mathrm{T}$ cell-mediated alloimmune response. Combination of agents that individually target signals 1,2 , and 3 is most common for maintenance immunosuppression to prevent acute rejection while minimizing toxicity associated with each medication. Induction immunosuppression at the time of transplant eliminates $\mathrm{T}$ lymphocytes from the circulation, thereby providing time to establish maintenance immunosuppression [24]. The different classes of immunosuppressive agents currently in clinical use are discussed below.

\section{Induction Agents}

Monoclonal antibodies that target the TCR (muromonabCD3) or CD52 (alemtuzumab) recognize and eliminate T lymphocytes from the circulation by activating complement, triggering apoptosis, or facilitating antibody-dependent cytotoxicity. These induce prolonged depletion of circulating $\mathrm{T}$ lymphocytes up to years after transplant. Antisera to human $\mathrm{T}$ lymphocytes or thymocytes such as horse antilymphocyte globulin or rabbit antithymocyte globulin contain polyclonal antibodies that recognize many different $\mathrm{T}$ cell antigens and cause lymphodepletion by mechanisms similar to that of the monoclonal antibodies. Basiliximab, an antibody specific for the IL-2 receptor (CD25), does not cause similar T cell depletion, but it has synergy with calcineurin inhibitors [25, 26].

\section{Maintenance Agents}

Corticosteroids have multiple immunosuppressive effects, including lympholysis at high doses, decreased production of inflammatory cytokines such as IL-1, IL-6, and TNF $\alpha$, and decreased expression of adhesion and costimulatory molecules necessary for antigen presentation. Myriad toxicities such as sodium and water retention, hypertension, adipose weight gain, hyperglycemia, dyslipidemia, and osteoporosis have led to the development of immunosuppressive protocols that spare or avoid corticosteroids. These protocols usually require induction with a lymphodepleting monoclonal antibody or polyclonal serum to minimize the risk of acute rejection [27, 28]. However, early corticosteroid withdrawal may not always be as effective in preventing acute rejection, as suggested by a retrospective analysis of a large cohort of liver transplant recipients [29].

Antimetabolites including azathioprine, mycophenolate mofetil, and leflunomide impair $\mathrm{T}$ and B lymphocyte proliferation by preventing de novo synthesis of adenosine, guanosine, and pyrimidines, respectively. This depletes the 
pool of nucleotides required for synthesis of RNA and DNA in rapidly dividing cells. In addition, azathioprine disrupts nucleic acid structure, and mycophenolate mofetil inhibits synthesis of cell adhesion molecules important for immune recognition. The use of these agents is primarily limited by myelotoxicity, including leucopenia, anemia, and thrombocytopenia.

Calcineurin inhibitors, including cyclosporine A and tacrolimus, are the most potent maintenance immunosuppressive agents. The introduction of cyclosporine in the early 1980 s revolutionized transplantation by dramatically improving renal, liver, and heart transplant survival. These agents inhibit the transcription of IL-2 messenger RNA, thereby blocking signal 3 of immune activation [30]. These agents serve as the primary agent of most maintenance immunosuppression. Tacrolimus and mycophenolate mofetil are thought to be the most effective combination therapy for prevention of acute rejection [31]. However, the utility of calcineurin inhibitors is limited by significant nephrotoxicity. This affects the long-term function of renal allografts and exacerbates native renal injury in the setting of other organ transplants, especially heart, liver, and lung.

Mammalian target of rapamycin (mTOR) inhibitors, including sirolimus and everolimus, prevent the progression of activated $\mathrm{T}$ lymphocytes into the cell cycle and therefore inhibit proliferation after signal 3 of immune activation [32]. Tacrolimus and sirolimus are synergistic and may be used together at lower doses than usual. mTOR inhibitors also block the proliferation of other cell types, including fibroblasts, epithelium, and endothelium, which can limit their use perioperatively due to impaired wound healing. This antiproliferative effect has been demonstrated to decrease the development of squamous cell skin cancer, the most common type of malignancy in solid organ transplant recipients.

Costimulatory blocking antibodies, the newest class of maintenance agents, inhibit signal 2 of immune activation. Belatacept, the first approved costimulatory blocker for kidney transplantation, is a chimeric molecule joining a human immunoglobulin heavy chain domain to CTLA4. CTLA-4, a molecule closely related in structure to $\mathrm{CD} 28$, is released from $\mathrm{T}$ lymphocyte intracellular stores after engagement of $\mathrm{CD} 28$ with its costimulatory ligand (CD80, CD86) on the APC. CTLA-4 binds to CD80 or CD86 with higher affinity than CD28 and inhibits signal 2. This agent is noninferior to cyclosporine A for de novo use renal transplants, promising long-term immunosuppression without nephrotoxicity [33, 34]. However, its use is limited by the need for monthly infusion and increased risk of post-transplant lymphoma, especially in nonimmune recipients of organs from donors exposed to
EBV virus, which is a strong risk factor for transformation of B lymphocytes.

No agents specific for B lymphocytes to prevent AMR are in clinical use. Anti-B lymphocyte therapy is confined to treatment of acute rejection. Human intravenous immune globulin (IVIG) is thought to modulate antibodyproducing cell activity through activation of the inhibitory Fc $\gamma$ III receptor, decreasing donor-specific antibody production [23, 35]. The monoclonal antibody rituximab, which targets the CD20 antigen expressed on B lymphocytes [25] and the proteasome inhibitor bortezomib, which targets active antibody-producing plasma cells [36], are indicated for treatment of B cell lymphoma and multiple myeloma, respectively. These agents deplete normal B lymphocytes and plasma cells along with malignant cells, resulting in decreased antibody concentrations. As a result, these have been adopted or use in a number of antibodymediated diseases, including acute and chronic antibodymediated allograft rejection.

\section{Clinical Immunosuppression Protocols for Solid Organ Transplant}

The choice of induction and maintenance immunosuppression depends on the type of organ transplant as well as recipient factors such as prior sensitization to HLA antigens and medical comorbidities. The intensity of immunosuppression required to prevent acute rejection varies among solid organ transplants, as seen in the likelihood of acute rejection for (in descending order) intestine, lung, heart, pancreas, kidney, and liver. In addition, simultaneous transplant of syngeneic solid organ transplants may diminish the immunogenicity of one or both of the grafts. This has been suggested to contribute to improved kidney survival in simultaneous liver-kidney transplant, especially in recipients who are sensitized to donor HLA antigens prior to transplant [37].

The liver may be less immunogenic due to several mechanisms. Hepatocyte shedding of soluble HLA molecules may cause $\mathrm{T}$ cell anergy by providing a sub-threshold signal 1. Cytokines may be absorbed extensively due to the large mass of the organ, thereby exhausting the immune response, and $\mathrm{T}$ cell anergy may occur by clonal exhaustion due to the presence of a large load of donor antigen [38]. Finally, mixed chimerism characterized by the exchange of mesenchymal stem cells [39], dendritic cells, or hematopoietic stem cells between the donor and the allograft has been proposed as a mechanism of tolerance induction to the allograft [40•*]. This may occur by the development of opposing recipient versus donor and donor versus host responses, leading to exhaustion of both responses. 
The most common protocols for kidney transplant employ triple maintenance therapy including a calcineurin inhibitor, an antimetabolite, and corticosteroids. The most common regimen includes tacrolimus, mycophenolate mofetil, and prednisone. Induction immunosuppression use varies among different centers. Although induction appears to decrease the risk of acute rejection, it has not been demonstrated to prolong long-term graft survival in comparison to protocols that do not use induction. Triple maintenance immunosuppression, often together with induction, is generally employed for heart, lung, pancreas, and intestinal transplant. Due to lower risk of rejection, liver transplant is managed by tapering off of the steroid and antimetabolite and continuing with tacrolimus monotherapy. Newer protocols will emerge with the development of novel classes of immunosuppressive drugs, with the emphasis on minimization of corticosteroids and calcineurin inhibitors to avoid their toxicities [41, 42].

\section{Alloimmune Response to Composite Tissue Transplants}

It was predicted that acute and chronic rejection would be more difficult to prevent and control than for VCA than for solid organ transplant, due primarily to the variable immunogenicity of specific tissues. Skin is highly immunogenic, and allogeneic skin transplant represents the most stringent experimental model of transplantation, thought in part due to the large number and variety of resident APCs [43]. It is therefore difficult a priori to predict the immunogenicity of a composite tissue allograft due to differing characteristics of allograft components. Nonetheless, hand and face transplant have been successfully performed using induction and maintenance immunosuppression employed for kidney transplant although at a higher level of dosing of immunosuppressive agents and higher rate of acute rejection $[44 \bullet, 45,46]$. The risk of acute rejection of skin when present in VCA may be affected by mechanisms similar to those operating in simultaneous liver-kidney or kidneypancreas transplantation.

The immunosuppressive protocols for composite tissue, including hand, face, and laryngeal transplant, have been modeled on that of induction with a lymphodepleting agent followed by triple maintenance therapy with tacrolimus, mycophenolate mofetil, and corticosteroids. The most variation in protocol use is seen in hand transplant, the most common form of composite tissue graft. Results of this immunosuppression protocol have in general been acceptable but at the cost of an increased risk of infectious complications, especially cytomegalovirus (CMV) infection. Different assays have been used to monitor for acute rejection, including skin punch biopsy and CT or MR angiography, but no highly sensitive and specific method has yet been identified.

\section{Laryngotracheal Transplantation}

The first clinically successful laryngeal transplant [3, 4•] used a protocol of OKT-3 induction and triple maintenance with cyclosporine, mycophenolate mofetil, and corticosteroids. Two episodes of acute rejection occurred. The first episode at 15 months after transplant was diagnosed based on decreased voice quality and was treated with pulse methylprednisolone and switch from cyclosporine to tacrolimus. The second episode occurred 6 years after transplant due to laboratory error in monitoring tacrolimus trough level and was manifested by laryngeal edema which resolved after tacrolimus trough level was restored to a therapeutic range. The graft was removed 14 years after transplant due to chronic rejection characterized by microvascular injury as well as atrophy and necrosis of cartilage. The second reported laryngeal transplant [5] employed a triple maintenance immunosuppression protocol including tacrolimus, leflunomide, and prednisone after rabbit antithymocyte globulin induction. Thus far, there has been no evidence of acute rejection. Of note, the recipient had been on maintenance immunosuppression for a functioning kidney-pancreas transplant. Leflunomide was used as an antimetabolite rather than mycophenolate mofetil due to polyoma virus nephropathy, because leflunomide has in vitro activity against polyoma virus while still providing immunosuppression.

\section{Facial Transplantation}

The first clinical full facial transplant was reported in 2005, and to date, 28 transplants have been performed [47••]. Immunosuppression follows protocols as described for solid organ transplantation, but the rate of acute rejection of facial allografts appears to be significantly higher, at $\sim 85 \%$. Histopathologic diagnosis is not yet as well established as for other types of transplant, especially for chronic rejection [48]. Current pathologic criteria for skin acute rejection include inflammation with lymphocytes, neutrophils, and eosinophils characterized as perivascular or interstitial infiltrates in the dermis or hypodermis with associated spongiosis, apoptosis, dyskeratosis, and necrosis of the epidermis and adnexa. Chronic rejection has not occurred as characterized by the chronic vascular intimal hyperplasia, hyalinosis, and luminal occlusion seen in other transplant types. This is of interest because chronic large vessel injury was identified without significant skin vasculopathy in one series of hand transplant recipients, thus calling into 
question the adequacy of usual screening for chronic rejection with skin punch biopsy and CT or MR angiography [49]. In facial transplant, the extensive vascular network supplying the skin may facilitate evaluation of large vessels for early diagnosis of vasculopathy. Furthermore, neovascularization in this network may mitigate the effect of vascular obliteration and end organ fibrosis, especially if the skin microvasculature is not as readily affected by chronic rejection as is the case for solid organ transplants.

\section{Induction of Immunologic Tolerance}

Although clinically successful, composite tissue transplantation has been complicated by increased risk of infection and by concern that the risk-benefit balance of chronic immunosuppression for non-life saving or extending therapy may not be favorable. This has prompted much interest in the development of pre-transplant conditioning protocols that induce durable immunologic tolerance to allografts without the need for chronic immunosuppression. Again, current thinking has been informed by the experience with such protocols used in solid organ transplantation. These regimens are based on the effect of mixed chimerism of hematopoietic stem cells or antigen-presenting cells between the donor and recipient. Multiple mechanisms may be involved. Donor antigen-presenting cells that have translocated to the recipient may render alloreactive $\mathrm{T}$ lymphocytes anergic. Donor hematopoietic stem cells (HSC) populate the bone marrow and generate $\mathrm{T}$ and $\mathrm{B}$ lymphocyte precursors exhibiting donor-specific antigens and develop into mature lymphocytes tolerant to donor antigens. Macrophage and dendritic cells of donor origin function during intrathymic development of $\mathrm{T}$ thymocytes to mediate selection and removal of donor-reactive cells before they develop into mature lymphocytes. Central regulatory $\mathrm{T}$ cells (Treg) generated in the thymus may also contribute to the intrathymic selection of nonreactive $\mathrm{T}$ lymphocytes, and peripheral Tregs may inhibit activation of alloreactive $T$ lymphocytes in the secondary lymphoid organs including lymph nodes and spleen.

Mixed bone marrow chimeras have been demonstrated in animal models to confer tolerance, but their creation requires high doses of pre-transplant radiation and severe bone marrow depletion to permit the engraftment of the donor stem cells. Many approaches to inducing tolerance in kidney transplantation have been developed with varying levels of success. The simultaneous stem cell transplant and kidney transplant from a live donor to a sibling with multiple myeloma and chronic kidney disease, along with post-transplant infusion of donor lymphocytes have resulted in durable graft tolerance in several patients [50-53]. Another protocol employing a "facilitator" cell population to ensure mixed bone marrow chimerism along with kidney transplant also appears to hold promise [54, 55].

The unique feature of multiple graft components, often including bone marrow, in composite tissue transplantation may allow exploitation of mixed bone marrow chimerism [56]. The identification of mechanisms that render skin less immunogenic in composite grafts may also provide key insights into tolerance induction.

\section{Conclusion}

The development of novel surgical techniques and immunosuppressive medications over the last 60 years has revolutionized the field of clinical transplantation. Successful immunosuppression protocols for solid organ transplant have served as the model for composite tissue transplantation. This strategy has led to the success of VCA and permitted the development of therapies that will continue to be more widely employed, including laryngeal transplantation. However, the risks of long-term immunosuppression, including malignancy, infection, and multiple drug-related toxicities remain barriers to the more widespread use of these therapies, and there is much interest in the development of specific therapies that induce allograftspecific tolerance without the risks of immunosuppression. Although routine use of clinical tolerance induction protocols remains in the future, the unique features of composite tissue transplantation make this an exciting area of research. The future indeed looks bright for the addition of head and neck transplantation to the therapeutic armamentarium of otolaryngology.

\section{Compliance with Ethics Guidelines}

Conflict of Interest Brian Gallay and Angelo DeMattos declare that they have no conflict of interest.

Human and Animal Rights and Informed Consent This article does not contain any studies with human or animal subjects performed by any of the authors.

\section{References}

Papers of particular interest, published recently, have been highlighted as:

- Of importance

•• Of major importance

1. Merrill JP, Murray JE, Hartwell H, Guild WR. Successful homotransplantation of the human kidney between identical twins. JAMA. 1956;160(4):277-82.

2. • Starzl TE. History of clinical transplantation. World J Surg. 2000;24(7):759-782. An older but excellent review of the history of transplantation by one of the pioneers in the field. 
3. Strome M, Stein J, Esclamado R, et al. Laryngeal transplantation and 40-month follow-up. N Eng J Med. 2001;344:1676-9.

4. - Knott PD, Hicks D, Braun W, Strome M. A 12-year perspective on the world's first total laryngeal transplant. Transplantation. 2011;91:804-805. Description of the long term outcome of the first total laryngeal transplant.

5. Farwell D, Birchall MA, Macchiarini P, et al. Laryngotracheal transplantation: technical modifications and functional outcomes. Laryngoscope. 2013;123:2502-8.

6. - Cruvinel Wde M, Mesquita D, Jr., Araujo JA, et al. Immune system - part I. Fundamentals of innate immunity with emphasis on molecular and cellular mechanisms of inflammatory response. Rev Bras Reumatol. 2010;50:434-61. Excellent detailed readable three part summary of immune system function. See also references 7 and 8 .

7. - Mesquita Junior D, Araujo JA, Catelan TT, et al. Immune system-part II: basis of the immunological response mediated by T and B lymphocytes. Rev Bras Reumatol. 2010;50:552-80. See reference 6 .

8. - de Souza AW, Mesquita Junior D, Araujo JA, et al. Immune system: part III. The delicate balance of the immune system between tolerance and autoimmunity. Rev Bras Reumatol. 2010;50:665-79. See reference 6.

9. Solez K, Colvin RB, Racusen LC, et al. Banff 07 classification of renal allograft pathology: updates and future directions. Am J Transpl. 2008;8:753-60.

10. Bhowmik DM, Dinda AK, Mahanta P, et al. The evolution of the Banff classification schema for diagnosing renal allograft rejection and its implications for clinicians. Indian $\mathrm{J}$ Nephrol. 2010;20:2-8.

11. Wyatt JI. Liver transplant pathology-messages for the nonspecialist. Histopathology. 2010;57:333-41.

12. Neil DA, Hubscher SG. Current views on rejection pathology in liver transplantation. Transpl Int. 2010;23:971-83.

13. Drachenberg CB, Odorico J, Demetris AJ, et al. Banff schema for grading pancreas allograft rejection: working proposal by a multidisciplinary international consensus panel. Am J Transpl. 2008;8:1237-49.

14. Stewart S, Winters GL, Fishbein MC, et al. Revision of the 1990 working formulation for the standardization of nomenclature in the diagnosis of heart rejection. J Heart Lung Transpl. 2005;24:1710-20.

15. Dark JH. Diagnosis of lung rejection. Lancet. 2004;363:1487-8.

16. Ruiz P, Bagni A, Brown R, et al. Histological criteria for the identification of acute cellular rejection in human small bowel allografts: results of the pathology workshop at the VIII International Small Bowel Transplant Symposium. Transpl Proc. 2004;36:335-7.

17. Cai J, Terasaki PI. Humoral theory of transplantation: mechanism, prevention, and treatment. Hum Immunol. 2005;66:334-42.

18. Clatworthy MR. Targeting B cells and antibody in transplantation. Am J Transpl. 2011;11:1359-67.

19. Sis B, Mengel M, Haas $\mathrm{M}$, et al. Banff '09 meeting report: antibody mediated graft deterioration and implementation of Banff working groups. Am J Transpl. 2010;10:464-71.

20. Mengel M, Sis B, Haas M, et al. Banff 2011 Meeting report: new concepts in antibody-mediated rejection. Am J Transpl. 2012;12:563-70.

21. Demetris AJ, Adeyi O, Bellamy CO, et al. Liver biopsy interpretation for causes of late liver allograft dysfunction. Hepatology. 2006;44:489-501.

22. Shilling RA, Wilkes DS. Immunobiology of chronic lung allograft dysfunction: new insights from the bench and beyond. Am J Transpl. 2009;9:1714-8.
23. Suzuki J, Isobe M, Morishita R, et al. Characteristics of chronic rejection in heart transplantation: important elements of pathogenesis and future treatments. Circ J. 2010;74:233-9.

24. Halloran PF. Immunosuppressive drugs for kidney transplantation. N Engl J Med. 2004;351:2715-29.

25. Mahmud N, Klipa D, Ahsan N. Antibody immunosuppressive therapy in solid-organ transplant: Part I. MAbs. 2010;2:148-56.

26. Klipa D, Mahmud N, Ahsan N. Antibody immunosuppressive therapy in solid organ transplant: Part II. MAbs. 2010;2:607-12.

27. Cantarovich D, Rostaing L, Kamar N, et al. Corticosteroid avoidance in adult kidney transplant recipients under rabbit antiT-lymphocyte globulin, mycophenolate mofetil and delayed cyclosporine microemulsion introduction. Transpl Int. 2010;23:313-24.

28. Garcia VD, Carvalho DB, Goncalves RT, et al. Randomized trial of early corticosteroid reduction vs. regular-dose corticosteroid maintenance in combination with tacrolimus and mycophenolate mofetil in living donor kidney transplant recipients: the Brazilian CORRETA trial. Clin Transpl. 2010;24:E109-15.

29. Meadows HB, Taber DJ, Pilch NA, et al. The impact of early corticosteroid withdrawal on graft survival in liver transplant recipients. Transpl Proc. 2012;44:1323-8.

30. Boyman O, Sprent J. The role of interleukin-2 during homeostasis and activation of the immune system. Nat Rev Immunol. 2012;12:180-90.

31. Dalal P, Shah G, Chhabra D, et al. Role of tacrolimus combination therapy with mycophenolate mofetil in the prevention of organ rejection in kidney transplant patients. Int J Nephrol Renovasc Dis. 2010;3:107-15.

32. Araki K, Ellebedy AH, Ahmed R. TOR in the immune system. Curr Opin Cell Biol. 2011;23:707-15.

33. Vincenti F, Charpentier B, Vanrenterghem Y, et al. A phase III study of belatacept-based immunosuppression regimens versus cyclosporine in renal transplant recipients (BENEFIT study). Am J Transpl. 2010;10:535-46.

34. Larsen CP, Grinyo J, Medina-Pestana J, et al. Belatacept-based regimens versus a cyclosporine A-based regimen in kidney transplant recipients: 2-year results from the BENEFIT and BENEFIT-EXT studies. Transplantation. 2010;90:1528-35.

35. Schwab I, Nimmerjahn F. Intravenous immunoglobulin therapy: how does IgG modulate the immune system? Nat Rev Immunol. 2013;13:176-89.

36. Lemy A, Toungouz M, Abramowicz D. Bortezomib: a new player in pre- and post-transplant desensitization? Nephrol Dial Transpl. 2010;25:3480-9.

37. Hanish SI, Samaniego M, Mezrich JD, et al. Outcomes of simultaneous liver/kidney transplants are equivalent to kidney transplant alone: a preliminary report. Transplantation. 2010;90: $52-60$.

38. Cunningham EC, Sharland AF, Bishop GA. Liver transplant tolerance and its application to the clinic: can we exploit the high dose effect? Clin Dev Immunol. 2013;2013:419692.

39. Wang $\mathrm{H}, \mathrm{Wu} \mathrm{M}$, Liu Y. Are mesenchymal stem cells major sources of safe signals in immune system? Cell Immunol. 2012;272:112-6.

40. $\bullet$ Starzl TE. Chimerism and tolerance in transplantation. Proc Natl Acad Sci USA. 2004;101 Suppl 2:14607-14. Although an older review, this provides an excellent introduction into the concept of immunologic tolerance by one of the pioneers of the field.

41. Webber A, Hirose R, Vincenti F. Novel strategies in immunosuppression: issues in perspective. Transplantation. 2011;91: 1057-64.

42. Halleck F, Friedersdorff F, Fuller TF, et al. New perspectives of immunosuppression. Transpl Proc. 2013;45:1224-31. 
43. Heath WR, Carbone FR. The skin-resident and migratory immune system in steady state and memory: innate lymphocytes, dendritic cells and T cells. Nat Immunol. 2013;14:978-85.

44. - Diaz-Siso JR, Bueno EM, Sisk GC et al. Vascularized composite tissue allotransplantation-state of the art. Clin Transpl. 2013;27:330-7. Excellent review of current status of vascularized composite tissue transplantation.

45. Brandacher G. Composite tissue transplantation. Methods Mol Biol. 1034;2013:103-15.

46. Elliott RM, Tintle SM, Levin LS. Upper extremity transplantation: current concepts and challenges in an emerging field. Curr Rev Musculoskelet Med. 2013;7(1):83-8.

47. •- Khalifian S, Brazio PS, Mohan R et al. Facial transplantation: the first 9 years. Lancet 2014. A current update on the outcomes of the first 28 facial transplants performed worldwide.

48. Cendales LC, Kanitakis J, Schneeberger S, et al. The Banff 2007 working classification of skin-containing composite tissue allograft pathology. Am J Transpl: Off J Am Soc Transpl Am Soc Transpl Surg. 2008;8:1396-400.

49. Kaufman CL, Ouseph R, Blair B, et al. Graft vasculopathy in clinical hand transplantation. Am J Transpl: Off J Am Soc Transpl Am Soc Transpl Surg. 2012;12:1004-16.
50. Andreola G, Chittenden M, Shaffer J, et al. Mechanisms of donorspecific tolerance in recipients of haploidentical combined bone marrow/kidney transplantation. Am J Transpl. 2011;11:1236-47.

51. Spitzer TR, Sykes M, Tolkoff-Rubin N, et al. Long-term followup of recipients of combined human leukocyte antigen-matched bone marrow and kidney transplantation for multiple myeloma with end-stage renal disease. Transplantation. 2011;91:672-6.

52. Kawai T, Sachs DH, Sykes M, et al. HLA-mismatched renal transplantation without maintenance immunosuppression. N Engl J Med. 2013;368:1850-2.

53. Scandling JD, Busque S, Dejbakhsh-Jones S, et al. Tolerance and withdrawal of immunosuppressive drugs in patients given kidney and hematopoietic cell transplants. Am J Transpl. 2012;12:1133-45.

54. Leventhal J, Abecassis M, Miller J, et al. Chimerism and tolerance without GVHD or engraftment syndrome in HLA-mismatched combined kidney and hematopoietic stem cell transplantation. Sci Transl Med. 2012;4:124ra28.

55. Markmann JF, Kawai T. The quest for transplantation tolerance: have we finally sipped from the cup? Sci Transl Med. 2012;4:124fs5.

56. Schneeberger S, Gorantla VS, Brandacher G, et al. Upperextremity transplantation using a cell-based protocol to minimize immunosuppression. Ann Surg. 2013;257:345-51. 